\title{
Montmorillonite K-10 as a catalyst in the synthesis of 5, 5-disubstituted hydantoins under ultrasound irradiation
}

\author{
J SAFARI* and L JAVADIAN \\ Laboratory of Organic Chemistry Research, Department of Organic Chemistry, College of Chemistry, \\ University of Kashan, P. O. Box: 87317-51167, Kashan, I.R. Iran \\ e-mail: Safari@kashanu.ac.ir
}

MS received 9 October 2012; revised 16 December 2012; accepted 21 January 2013

\begin{abstract}
The rapid and highly efficient synthesis of 5,5-disubstituted hydantoins achieved by threecomponent condensation of corresponding ketones and aldehydes, potassium cyanide and ammonium carbonate in an additional reaction under ultrasound irradiation by using montmorillonite $\mathrm{K}-10$ as a heterogenous catalyst. Utilizing ultrasound irradiation and montmorillonite in this method has several benefits, for example short reaction time, high yield and use of recyclable catalyst.
\end{abstract}

Keywords. Ultrasound irradiation; 5,5-disubstituted hydantoin; montmorillonite K-10; Bucherer-Bergs reaction.

\section{Introduction}

Hydantoins (2,4-imidazolidine-diones) are an important class of heterocycles. ${ }^{1}$ Hydantoin nucleus is a common 5-membered ring containing a reactive cyclic urea core. ${ }^{2}$ Imidazolidine-diones are a class of bioactive molecules that have broad medicinal, ${ }^{3}$ antiulcers, ${ }^{4}$ anticonvulsants, ${ }^{5}$ anti-tumour, ${ }^{6}$ antiarrhythmic,${ }^{7}$ anticancer, ${ }^{8}$ antimicrobial, ${ }^{9}$ antidiabetic, ${ }^{10}$ and agrochemical (herbicidal and fungicidal) applications. ${ }^{11}$

Hydantoin derivatives are synthetically valuable, for e.g., as precursors to amino acid and pyruvic acid derivatives. ${ }^{12,13}$ Several methods are used for synthesis of 5,5-disubstituted hydantoins. ${ }^{14-17}$ The BuchererBergs synthesis is most commonly used for preparation of these heterocycles. It is multi-component reaction that yields hydantoins from the reaction of ketone (or aldehyde) with cyanide, ammonia and carbon dioxide or ammonium carbonate. ${ }^{18}$ This reaction used in the preparation of 5,5-disubstituted hydantoin derivatives suffers from drawbacks such as low yield and long reaction time.

Consequently, it is desirable to develop an efficient and simple method to synthesize 5,5-disubstituted hydantoins. Catalysts are very useful in achieving this goal. In recent years, there has been considerable growth of interest in the catalysis of organic reactions by clays. Clays as heterogenous catalysts are helpful to the synthetic chemist in a big way. By-products can

*For correspondence be avoided or minimized by these solid acids. Using montmorillonite $\mathrm{K}-10$ as an economically solid catalyst has several advantages such as ease of handling, being inexpensive and mild reaction conditions. ${ }^{19-21}$

On the other hand, use of ultrasound irradiation for the optimization and acceleration of organic reactions has rapidly increased. ${ }^{22-24}$ The combined use of ultrasound reduced the reaction time significantly. High local temperature and pressure produced by cavitation lead to a diverse set of applications of ultrasound such as accelerating the rate of reaction, changing the reaction pathway, enhancing chemical reactivity and important uses in synthetic organic compounds. ${ }^{25,26}$

This prompted us to study the possibility of preparation of 5,5-disubstituted hydantions using the Bucherer-Bergs method involving montmorillonite K-10 catalysis and utilizing ultrasound irradiation.

\section{Experimental}

\subsection{Materials and instruments}

In a typical procedure, chemicals were purchased from Merck chemical company. ${ }^{1} \mathrm{NMR}(400 \mathrm{MHz})$ and ${ }^{13} \mathrm{C}$ NMR $(100 \mathrm{MHz})$ spectra were recorded on a Bruker DPX-400 Avance Spectrometer. Tetramethyl silane (TMS) was used as an internal reference. IR spectra were obtained on a Magna-550 Nicolet instrument. Vibrational transition frequencies were reported 
as wave numbers $\left(\mathrm{cm}^{-1}\right)$, and band intensities designated as weak $(\mathrm{w})$, medium $(\mathrm{m})$ and strong $(\mathrm{s})$. Sonication was performed in a UP $400 \mathrm{~S}$ ultrasonic processor equipped with a $3 \mathrm{~mm}$ wide and $140 \mathrm{~mm}$ long probe, which was immersed directly into the reaction mixture. The operating frequency was $50 \mathrm{KHz}$ and the output power was $0-400 \mathrm{~W}$ through manual adjustment. UV spectra were recorded on a Hitachi 200-20 spectrometer using spectrophotometric grade ethanol (Baker). Melting points were obtained with a micro melting point apparatus (Electrothermal, MK3) and are uncorrected. The characteristics of montmorillonite K-10 are: surface area $=220-270 \mathrm{~m}^{2} \mathrm{~g}^{-1}$, bulk density $=300-370 \mathrm{~g}$ $1^{-1}$, specific gravity $=2.5 \mathrm{~g} \mathrm{ml}$, refractive index $=1.51$, crystal system, monoclinic.

\subsection{General procedure for the synthesis of hydantoin derivatives involving montmorillonite $K-10$ under ultrasonic irradiation}

To a solution of $1 \mathrm{mmol}$ of aldehyde or ketone, $1.3 \mathrm{mmol}$ potassium cyanide, $6 \mathrm{mmol}$ ammonium carbonate in $5 \mathrm{~mL}$ EtOH and $5 \mathrm{~mL}$ of $\mathrm{H}_{2} \mathrm{O}, 100 \mathrm{mg}$ montmorillonite $\mathrm{K}-10$ was added and the mixture was exposed to ultrasonic irradiation at $45^{\circ} \mathrm{C}$ for the period as indicated in table 1 . Progress of the reaction was monitored by TLC (petroleum ether: ethyl acetate,
$1: 1.2 \mathrm{v} / \mathrm{v})$. The catalyst was removed by filtration and washed with pentane. Then, the reaction mixture was neutralized with diluted hydrochloric acid and filtered. The solid product obtained was washed with water and recrystallized from ethanol. Pure product was obtained in good yield (scheme 1). The structure of these compounds has been investigated using different methods of spectroscopy and spectrometry: UV, ${ }^{1} \mathrm{H}$ NMR, ${ }^{13} \mathrm{C}$ NMR, IR.

\subsection{Spectral data of 5,5-disubstituted hydantoins}

2.3a 5-Phenyl imidazolidine-2,4-dione $\left(\mathrm{C}_{9} \mathrm{H}_{8} \mathrm{~N}_{2} \mathrm{O}_{2}\right.$, $4 a)$ : White powdery crystals; UV $\left(\mathrm{CH}_{3} \mathrm{OH}\right) \lambda_{\max }$ : $284 \mathrm{~nm} ;{ }^{1} \mathrm{H}$ NMR (DMSO- $\left.d_{6}, 400 \mathrm{MHz}\right) \delta: 10.79$ (s, $\left.1 \mathrm{H}, \mathrm{N}_{3}-\mathrm{H}\right), 8.41\left(\mathrm{~s}, 1 \mathrm{H}, \mathrm{N}_{1}-\mathrm{H}\right), 7.31(\mathrm{t}, J=6.8 \mathrm{~Hz}$, $1 \mathrm{H}), 7.35(\mathrm{~d}, J=6.89 \mathrm{~Hz}, 2 \mathrm{H}), 7.40(\mathrm{t}, J=6.89 \mathrm{~Hz}$, $2 \mathrm{H}), 5.16(\mathrm{~s}, 1 \mathrm{H}) ;{ }^{13} \mathrm{C}$ NMR (DMSO- $\left.d_{6}, 100 \mathrm{MHz}\right) \delta$ : $173.77\left(\mathrm{C}_{4}=\mathrm{O}\right), 157.67\left(\mathrm{C}_{2}=\mathrm{O}\right), 136.49(\mathrm{C}), 129.09$ (2CH), $128.77(2 \mathrm{CH}), 127.11(\mathrm{CH}), 62.26\left(\mathrm{C}_{\text {spiro }}\right)$; IR $\left(\mathrm{KBr} \mathrm{cm}^{-1}\right) \bar{v}: 3500(\mathrm{~N}-\mathrm{H}, \mathrm{s}), 3300(\mathrm{~N}-\mathrm{H}, \mathrm{s}), 3150$ $(=\mathrm{C}-\mathrm{H}), 3024(=\mathrm{C}-\mathrm{H}), 1745(\mathrm{C}=\mathrm{O}), 1720(\mathrm{C}=\mathrm{O})$, $1450(\mathrm{C}=\mathrm{C}), 1420(\mathrm{~N}-\mathrm{H}, \mathrm{b})$.

2.3b 5-(4-Methylphenyl)-imidazolidine-2,4-dione $\left(\mathrm{C}_{10} \mathrm{H}_{10} \mathrm{~N}_{2} \mathrm{O}_{2}, 4 \boldsymbol{b}\right)$ : White crystal; UV $\left(\mathrm{CH}_{3} \mathrm{OH}\right) \lambda_{\max }$ : $276 \mathrm{~nm} ;{ }^{1} \mathrm{H}$ NMR (DMSO- $\left.d_{6}, 400 \mathrm{MHz}\right) \delta: 10.74$ (s,

Table 1. Montmorillonite K-10 catalysed synthesis of 5,5-disubstituted hydantoins under ultrasonic irradiation.

\begin{tabular}{|c|c|c|c|c|c|c|}
\hline Entry & $\mathrm{R}^{1}$ & $\mathrm{R}^{2}$ & Product & Time (min)/yield (\%) & m.p. $\left({ }^{\circ} \mathrm{C}\right)$ Rep. & m.p. $\left({ }^{\circ} \mathrm{C}\right)$ Lit. \\
\hline A & $\mathrm{H}$ & $\mathrm{C}_{6} \mathrm{H}_{5}$ & $4 \mathbf{a}$ & $16 / 92$ & $164-165$ & $163^{12}$ \\
\hline B & $\mathrm{H}$ & $\mathrm{p}-\mathrm{CH}_{3} \mathrm{C}_{6} \mathrm{H}_{5}$ & $4 b$ & $20 / 94$ & $183-184$ & $182.5^{27}$ \\
\hline $\mathrm{C}$ & $\mathrm{H}$ & $\mathrm{p}-\mathrm{OCH}_{3} \mathrm{C}_{6} \mathrm{H}_{5}$ & $4 c$ & $26 / 88$ & 193-197 & - \\
\hline $\mathrm{D}$ & $\mathrm{H}$ & $\mathrm{o}-\mathrm{OCH}_{3} \mathrm{C}_{6} \mathrm{H}_{5}$ & $4 d$ & $28 / 90$ & $183-185$ & $186-187^{27}$ \\
\hline $\mathrm{E}$ & $\mathrm{H}$ & $\mathrm{o}-\mathrm{ClC}_{6} \mathrm{H}_{5}$ & $4 e$ & $19 / 96$ & $178-179$ & $176^{27}$ \\
\hline $\mathrm{F}$ & $\mathrm{H}$ & & $4 f$ & $18 / 94$ & $259-262$ & $258-261^{14}$ \\
\hline G & $\mathrm{H}$ & & $4 g$ & 20/94 & $148-149$ & $147^{27}$ \\
\hline $\mathrm{H}$ & $\mathrm{CH}_{3}$ & $\mathrm{i}-\mathrm{Bu}$ & $4 \mathrm{~h}$ & $32 / 98$ & $148-150$ & $148^{27}$ \\
\hline I & $\mathrm{R}=$ & & $4 i$ & $30 / 98$ & 219-221 & $220^{12}$ \\
\hline $\mathrm{J}$ & $\mathrm{CH}_{3}$ & $\mathrm{C}_{6} \mathrm{H}_{5}$ & $4 j$ & $30 / 87$ & 197-199 & $195-196^{28}$ \\
\hline K & $\mathrm{CH}_{3}$ & $\mathrm{p}-\mathrm{ClC}_{6} \mathrm{H}_{4}$ & $4 k$ & $31 / 95$ & $261-262$ & $262-263^{29}$ \\
\hline 1 & $\mathrm{CH}_{3}$ & $\mathrm{~m}-\mathrm{CH}_{3} \mathrm{C}_{6} \mathrm{H}_{4}$ & 41 & $50 / 97$ & $180-182$ & $175-180^{28}$ \\
\hline M & $\mathrm{CH}_{3}$ & $\mathrm{~m}-\mathrm{ClC}_{6} \mathrm{H}_{4}$ & $4 m$ & 28/95 & $180-183$ & $180-182^{28}$ \\
\hline $\mathrm{N}$ & $\mathrm{C}_{6} \mathrm{H}_{5}$ & $\mathrm{C}_{6} \mathrm{H}_{5}$ & $4 n$ & $72 / 86$ & $295-296$ & $297-298^{30}$ \\
\hline $\mathrm{O}$ & & & 40 & $82 / 85$ & $300>$ & $324-325^{27}$ \\
\hline
\end{tabular}




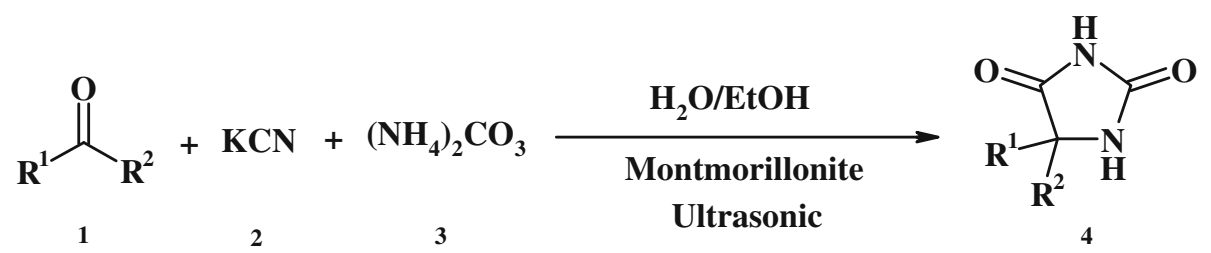

Scheme 1. Synthesis of hydantoin derivatives by using monmorillonite K-10 under ultrasonic irradiation.

$\left.1 \mathrm{H}, \mathrm{N}_{3}-\mathrm{H}\right), 8.35$ (s, 1H, $\left.\mathrm{N}_{1}-\mathrm{H}\right), 7.19$ (s, 4H), 5.09 (s, $1 \mathrm{H}), 2.29(\mathrm{~s}, 3 \mathrm{H}) ;{ }^{13} \mathrm{C}$ NMR (DMSO- $\left.d_{6}, 100 \mathrm{MHz}\right) \delta$ : $174.82(\mathrm{C}=\mathrm{O}), 157.97(\mathrm{C}=\mathrm{O}), 138.01(\mathrm{C}), 133.57$ $(2 \mathrm{CH}), 129.64(2 \mathrm{CH}), 127.08(\mathrm{C}), 61.46(\mathrm{C}-\mathrm{H}), 21.15$ $(\mathrm{C}-\mathrm{H})$; IR $\left(\mathrm{KBr} \mathrm{cm}^{-1}\right) \bar{v}: 3524(\mathrm{~N}-\mathrm{H}, \mathrm{s}), 3426(\mathrm{~N}-\mathrm{H}$, s), $3050(=\mathrm{C}-\mathrm{H}), 2970(\mathrm{C}-\mathrm{H}), 1777(\mathrm{C}=\mathrm{O}), 1755$ $(\mathrm{C}=\mathrm{O}), 1500(\mathrm{C}=\mathrm{C}), 1448(\mathrm{~N}-\mathrm{H}, \mathrm{b})$.

2.3c 5-(4-Methoxyphenyl)-imidazolidine-2,4-dione $\left(\mathrm{C}_{10} \mathrm{H}_{10} \mathrm{~N}_{2} \mathrm{O}_{3}, 4 c\right)$ : White needles; UV $\left(\mathrm{CH}_{3} \mathrm{OH}\right)$ $\lambda_{\max }: 280 \mathrm{~nm} ;{ }^{1} \mathrm{H}$ NMR (DMSO- $\left.d_{6}, 400 \mathrm{MHz}\right) \delta: 9.3$ (s, $\left.1 \mathrm{H}, \mathrm{N}_{3}-\mathrm{H}\right), 8.34\left(\mathrm{~s}, 1 \mathrm{H}, \mathrm{N}_{1}-\mathrm{H}\right), 7.21(\mathrm{~d}, J=7.7 \mathrm{~Hz}$, $2 \mathrm{H}), 6.94(\mathrm{~d}, J=7.7 \mathrm{~Hz}, 2 \mathrm{H}), 5.08(\mathrm{~s}, 1 \mathrm{H}), 3.74(\mathrm{~s}$, $3 \mathrm{H}) ;{ }^{13} \mathrm{C}$ NMR (DMSO- $\left.d_{6}, 100 \mathrm{MHz}\right) \delta: 166.71(\mathrm{C}=$ $\mathrm{O}), 160.04(\mathrm{C}=\mathrm{O}), 158.37(\mathrm{C}), 127.12(2 \mathrm{CH}), 120.03$ $(2 \mathrm{CH}), 113.63(\mathrm{C}), 61.04\left(\mathrm{C}_{\text {spiro }}\right), 55.20(\mathrm{C}-\mathrm{H})$; IR $\left(\mathrm{KBr} \mathrm{cm}^{-1}\right) \bar{v}: 3350(\mathrm{~N}-\mathrm{H}, \mathrm{m}), 3215(\mathrm{~N}-\mathrm{H}, \mathrm{s}), 1779$ $(\mathrm{C}=\mathrm{O}, \mathrm{s}), 1732(\mathrm{C}=\mathrm{O}, \mathrm{s}), 1610(\mathrm{C}=\mathrm{C}, \mathrm{w}), 1515$ $(\mathrm{C}=\mathrm{C}, \mathrm{w}), 1246(\mathrm{C}-\mathrm{O}, \mathrm{m})$.

2.3d 5-2-Methoxyphenyl)-imidazolidine-2,4-dione $\left(\mathrm{C}_{10} \mathrm{H}_{10} \mathrm{~N}_{2} \mathrm{O}_{3}, 4 d\right)$ : White needles; UV $\left(\mathrm{CH}_{3} \mathrm{OH}\right) \lambda_{\max }$ : $274 \mathrm{~nm} ;{ }^{1} \mathrm{H}$ NMR (DMSO- $\left.d_{6}, 400 \mathrm{MHz}\right) \delta: 9.40$ (s, $1 \mathrm{H}$, $\left.\mathrm{N}_{3}-\mathrm{H}\right), 7.99$ (s, 1H, N $\left.1-\mathrm{H}\right), 7.30(\mathrm{~d}, J=7.00 \mathrm{~Hz}, 1 \mathrm{H})$, $7.08-6.92(\mathrm{~m}, 2 \mathrm{H}), 6.85(\mathrm{~d}, J=7.00 \mathrm{~Hz}, 1 \mathrm{H}), 4.85$ (s, $1 \mathrm{H}), 4.01(\mathrm{~s}, 3 \mathrm{H}) ;{ }^{13} \mathrm{C}$ NMR (DMSO- $\left.d_{6}, 100 \mathrm{MHz}\right) \delta$ : $166.01(\mathrm{C}=\mathrm{O}), 161.12(\mathrm{C}=\mathrm{O}), 157.62(\mathrm{C}), 128.13$ $(2 \mathrm{CH}), 119.11(2 \mathrm{CH}), 114.02(\mathrm{C}), 62.01\left(\mathrm{C}_{\text {spiro }}\right), 54.17$ $(\mathrm{C}-\mathrm{H})$; IR $\left(\mathrm{KBr} \mathrm{cm}^{-1}\right) \bar{v}: 3348(\mathrm{~N}-\mathrm{H}, \mathrm{m}), 3217(\mathrm{~N}-\mathrm{H}$, s), $1780(\mathrm{C}=\mathrm{O}, \mathrm{s}), 1729(\mathrm{C}=\mathrm{O}, \mathrm{s}), 1612(\mathrm{C}=\mathrm{C}, \mathrm{w})$, $1518(\mathrm{C}=\mathrm{C}, \mathrm{w}), 1250(\mathrm{C}-\mathrm{O}, \mathrm{m})$.

2.3e 5-(2-Chlorophenyl)-imidazolidine-2,4-dione $\left(\mathrm{C}_{9} \mathrm{H}_{7} \mathrm{~N}_{2} \mathrm{O}_{2} \mathrm{Cl}, 4 \boldsymbol{4}\right)$ : White needles; UV $\left(\mathrm{CH}_{3} \mathrm{OH}\right)$ $\lambda_{\max }: 275 \mathrm{~nm} ;{ }^{1} \mathrm{H}$ NMR (DMSO- $\left.d_{6}, 400 \mathrm{MHz}\right) \delta$ : $9.70\left(\mathrm{~s}, 1 \mathrm{H}, \mathrm{N}_{3}-\mathrm{H}\right), 8.03\left(\mathrm{~s}, 1 \mathrm{H}, \mathrm{N}_{1}-\mathrm{H}\right), 7.90(\mathrm{~d}$, $J=6.98 \mathrm{~Hz}, 1 \mathrm{H}), 7.71(\mathrm{t}, J=6.98 \mathrm{~Hz}, 1 \mathrm{H}), 7.35-$ $7.36(\mathrm{~m}, 2 \mathrm{H}), 5.13(\mathrm{~s}, 1 \mathrm{H}) ;{ }^{13} \mathrm{C}$ NMR (DMSO- $d_{6}$, $100 \mathrm{MHz}) \delta: 168.71(\mathrm{C}=\mathrm{O}), 151.04(\mathrm{C}=\mathrm{O}), 158.37$ (C), $127.12(2 \mathrm{CH}), 120.03(2 \mathrm{CH}), 113.63(\mathrm{C}), 61.04$ $\left(\mathrm{C}_{\text {spiro }}\right)$; IR $\left(\mathrm{KBr} \mathrm{cm}^{-1}\right) \bar{\nu}: 3350(\mathrm{~N}-\mathrm{H}, \mathrm{m}), 3215(\mathrm{~N}-\mathrm{H}$, $\mathrm{s}), 1779(\mathrm{C}=\mathrm{O}, \mathrm{s}), 1732(\mathrm{C}=\mathrm{O}, \mathrm{s}), 1613(\mathrm{C}=\mathrm{C}, \mathrm{w})$, $1514(\mathrm{C}=\mathrm{C}, \mathrm{w})$.
$2.3 \mathrm{f}$ 5-(3-Pyrrolyl)-imidazolidine-2,4-dione $\left(\mathrm{C}_{7} \mathrm{H}_{6} \mathrm{~N}_{3} \mathrm{O}_{2}\right.$, $4 f)$ : White needles; UV $\left(\mathrm{CH}_{3} \mathrm{OH}\right) \lambda_{\max }: 280 \mathrm{~nm} ;{ }^{1} \mathrm{H}$ NMR (DMSO- $\left.d_{6}, 400 \mathrm{MHz}\right) \delta: 12.02\left(\mathrm{~s}, 1 \mathrm{H}, \mathrm{N}_{3}-\mathrm{H}\right)$, $10.58\left(\mathrm{~s}, 1 \mathrm{H}, \mathrm{N}_{1}-\mathrm{H}\right), 8.06(\mathrm{~s}, 1 \mathrm{H}), 7.59(\mathrm{~s}, 1 \mathrm{H}), 7.09(\mathrm{~s}$, $1 \mathrm{H}), 4.99(\mathrm{~s}, 1 \mathrm{H}) ;{ }^{13} \mathrm{C}$ NMR (DMSO- $\left.d_{6}, 100 \mathrm{MHz}\right) \delta$ : $167.01(\mathrm{C}=\mathrm{O}), 159.21(\mathrm{C}=\mathrm{O}), 130.99(\mathrm{CH}), 122.27$ (1CH), $118.07(2 \mathrm{CH}), 113.70(\mathrm{C}), 62.00\left(\mathrm{C}_{\text {spiro }}\right)$; IR $\left(\mathrm{KBr} \mathrm{cm}^{-1}\right) \bar{v}: 3400(\mathrm{~N}-\mathrm{H}, \mathrm{s}), 3351(\mathrm{~N}-\mathrm{H}, \mathrm{m}), 3219$ $(\mathrm{N}-\mathrm{H}, \mathrm{s}), 1780(\mathrm{C}=\mathrm{O}, \mathrm{s}), 1735(\mathrm{C}=\mathrm{O}, \mathrm{s}), 1612(\mathrm{C}=$ $\mathrm{C}, \mathrm{w}), 1517(\mathrm{C}=\mathrm{C}, \mathrm{w})$.

$2.3 \mathrm{~g}$ 5-(2-Furyl)-imidazolidine-2,4-dione $\left(\mathrm{C}_{7} \mathrm{H}_{6} \mathrm{~N}_{2} \mathrm{O}_{3}\right.$, $4 \boldsymbol{g})$ : White needles; UV $\left(\mathrm{CH}_{3} \mathrm{OH}\right) \lambda_{\max }: 273 \mathrm{~nm} ;{ }^{1} \mathrm{H}$ NMR (DMSO- $\left.d_{6}, 400 \mathrm{MHz}\right) \delta: 9.40\left(\mathrm{~s}, 1 \mathrm{H}, \mathrm{N}_{3}-\mathrm{H}\right)$, $9.61\left(\mathrm{~s}, 1 \mathrm{H}, \mathrm{N}_{1}-\mathrm{H}\right), 7.61(\mathrm{~s}, 1 \mathrm{H}), 6.85(\mathrm{~d}, J=3.2 \mathrm{~Hz}$, $1 \mathrm{H}), 5.97(\mathrm{~d}, J=3.2,1 \mathrm{H}), 4.71(\mathrm{~s}, 1 \mathrm{H}) ;{ }^{13} \mathrm{C}$ NMR $\left(\mathrm{DMSO}-d_{6}, 100 \mathrm{MHz}\right) \delta$ : $167.61(\mathrm{C}=\mathrm{O}), 162.13(\mathrm{C}=$ O), $142.19(\mathrm{C}), 118.02(1 \mathrm{CH}), 117.16(1 \mathrm{CH}), 114.05$ (1CH), $62.17\left(\mathrm{C}_{\text {spiro }}\right)$; IR $\left(\mathrm{KBr} \mathrm{cm}^{-1}\right) \bar{\nu}: 3400(\mathrm{~N}-\mathrm{H}, \mathrm{m})$, $3217(\mathrm{~N}-\mathrm{H}, \mathrm{s}), 1781(\mathrm{C}=\mathrm{O}, \mathrm{s}), 1742(\mathrm{C}=\mathrm{O}, \mathrm{s}), 1623$ $(\mathrm{C}=\mathrm{C}, \mathrm{w}), 1512(\mathrm{C}=\mathrm{C}, \mathrm{w})$.

2.3h 5-Isobutyl-5-methyl-imidazolidine-2,4-dione $\left(\mathrm{C}_{8} \mathrm{H}_{14} \mathrm{~N}_{2} \mathrm{O}_{2}\right.$, 4h): White needles; UV $\left(\mathrm{CH}_{3} \mathrm{OH}\right)$ $\lambda_{\max }: 312 \mathrm{~nm} ;{ }^{1} \mathrm{H}$ NMR (DMSO- $\left.d_{6}, 400 \mathrm{MHz}\right) \delta: 10.52$ $\left(\mathrm{s}, 1 \mathrm{H}, \mathrm{N}_{3}-\mathrm{H}\right), 7.89\left(\mathrm{~s}, 1 \mathrm{H}, \mathrm{N}_{1}-\mathrm{H}\right), 1.57-1.51(\mathrm{~m}$, $2 \mathrm{H}), 1.46-1.42(\mathrm{~m}, 1 \mathrm{H}), 1.20(\mathrm{~s}, 3 \mathrm{H}), 0.87-0.86(\mathrm{~d}$, $J=5 \mathrm{~Hz}, 3 \mathrm{H}), 0.79-0.77(\mathrm{~d}, J=5 \mathrm{~Hz}, 3 \mathrm{H}) ;{ }^{13} \mathrm{C}$ NMR(DMSO- $\left.d_{6}, 100 \mathrm{MHz}\right) \delta: 166.46(\mathrm{C}=\mathrm{O}), 158.47$ $(\mathrm{C}=\mathrm{O}), 67.16\left(\mathrm{C}_{\text {spiro }}\right), 46.67(\mathrm{C}-\mathrm{H}), 23.99(\mathrm{C}-\mathrm{H})$, $22.17(\mathrm{C}-\mathrm{H}), 20.92(\mathrm{C}-\mathrm{H})$; $\mathrm{IR}\left(\mathrm{KBr} \mathrm{cm}{ }^{-1}\right) \bar{v}: 3197$ (N-H, s), 3117 (N-H, s), $2981(\mathrm{C}-\mathrm{H}), 2971(\mathrm{C}-\mathrm{H})$, $1771(\mathrm{C}=\mathrm{O}), 1720(\mathrm{C}=\mathrm{O}), 1403(\mathrm{~N}-\mathrm{H}, \mathrm{b}), 1195$.

2.3i 1,3-Diazaspiro[4,5]decane-2,4-dione $\left(\mathrm{C}_{8} \mathrm{H}_{12} \mathrm{~N}_{2} \mathrm{O}_{2}\right.$, $4 i)$ : White needles; UV $\left(\mathrm{CH}_{3} \mathrm{OH}\right) \lambda_{\max }: 348 \mathrm{~nm} ;{ }^{1} \mathrm{H}$ NMR (DMSO- $\left.d_{6}, 400 \mathrm{MHz}\right) \delta: 10.47\left(\mathrm{~s}, 1 \mathrm{H}, \mathrm{N}_{3}-\mathrm{H}\right)$, $8.38\left(\mathrm{~s}, 1 \mathrm{H}, \mathrm{N}_{1}-\mathrm{H}\right), 1.62-1.45(\mathrm{~m}, 9 \mathrm{H}), 1.21-1.27(\mathrm{~m}$, $1 \mathrm{H}) . ;{ }^{13} \mathrm{C}$ NMR (DMSO- $\left.d_{6}, 100 \mathrm{MHz}\right) \delta: 179.61(\mathrm{C}=$ $\mathrm{O}), 157.51(\mathrm{C}=\mathrm{O}), 62.89\left(\mathrm{C}_{\text {spiro }}\right), 39.76\left(2 \mathrm{CH}_{2}\right), 25.33$ $\left(2 \mathrm{CH}_{2}\right), 21.71\left(\mathrm{CH}_{2}\right)$; IR $\left(\mathrm{KBr} \mathrm{cm}{ }^{-1}\right) \bar{v}: 3250(\mathrm{~N}-\mathrm{H}$,$) ,$ 3199 (N-H, s), 3069 (=C-H, s), 2936 (C-H, s), 1776 
$(\mathrm{C}=\mathrm{O}, \mathrm{m}), 1734(\mathrm{C}=\mathrm{O}, \mathrm{s}), 1456(\mathrm{~N}-\mathrm{H}, \mathrm{b}, \mathrm{s}), 1411$ (s), 1292 (m), 1228 (m), 1072 (m), 942 (m), 780 (m), $753(\mathrm{~m})$.

2.3j 5-Methyl-5-phenyl-imidazolidine-2,4-dione $\left(\mathrm{C}_{10} \mathrm{H}_{10} \mathrm{~N}_{2} \mathrm{O}_{2}, 4 \mathrm{j}\right)$ : White needles; UV $\left(\mathrm{CH}_{3} \mathrm{OH}\right)$ $\lambda_{\max }: 252 \mathrm{~nm} ;{ }^{1} \mathrm{H}$ NMR (DMSO- $\left.d_{6}, 400 \mathrm{MHz}\right) \delta$ : 10.75 (s, $\left.1 \mathrm{H}, \mathrm{N}_{3}-\mathrm{H}\right), 8.62$ (s, $\left.1 \mathrm{H}, \mathrm{N}_{1}-\mathrm{H}\right), 7.47-7.45$ (d, $J=6 \mathrm{~Hz}, 2 \mathrm{H}), 7.40-7.36(\mathrm{t}, J=6 \mathrm{~Hz}, 2 \mathrm{H}), 7.32-7.31$ $(\mathrm{t}, J=6 \mathrm{~Hz}, 1 \mathrm{H}), 1.64(\mathrm{~s}, 3 \mathrm{H}) ;{ }^{13} \mathrm{C}$ NMR (DMSO- $d_{6}$, $100 \mathrm{MHz}) \delta: 166.16(\mathrm{C}=\mathrm{O}), 157.41(\mathrm{C}=\mathrm{O}), 127.41$ (C), $127.38(2 \mathrm{CH}), 126.79(2 \mathrm{CH}), 123.70(2 \mathrm{CH})$, $71.22\left(\mathrm{C}_{\text {spiro }}\right), 22.14(\mathrm{C}-\mathrm{H})$; IR $\left(\mathrm{KBr} \mathrm{cm}^{-1}\right) \bar{v}: 3282$ $(\mathrm{N}-\mathrm{H}, \mathrm{s}), 3208(\mathrm{~N}-\mathrm{H}, \mathrm{s}), 3064(=\mathrm{C}-\mathrm{H}, \mathrm{m}), 2989(\mathrm{C}-\mathrm{H}$, $\mathrm{m}), 1731(\mathrm{C}=\mathrm{O}, \mathrm{s}), 1726(\mathrm{C}=\mathrm{O}, \mathrm{s}), 787(=\mathrm{C}-\mathrm{H}, \mathrm{b})$, $482(\mathrm{w})$.

2.3k 5-(4-Chlorophenyl)-5-methyl-imidazolidine-2,4dione $\left(\mathrm{C}_{10} \mathrm{H}_{9} \mathrm{ClN}_{2} \mathrm{O}_{2}, 4 \boldsymbol{k}\right)$ : White needles; UV $\left(\mathrm{CH}_{3} \mathrm{OH}\right) \quad \lambda_{\max }: 272 \mathrm{~nm} ;{ }^{1} \mathrm{H} \quad \mathrm{NMR}$ (DMSO- $d_{6}$, $400 \mathrm{MHz}) \delta: 10.6\left(\mathrm{~s}, 1 \mathrm{H}, \mathrm{N}_{3}-\mathrm{H}\right), 8.64\left(\mathrm{~s}, 1 \mathrm{H}, \mathrm{N}_{1}-\mathrm{H}\right)$, $7.49(\mathrm{~d}, J=8.3,2 \mathrm{H}), 7.47(\mathrm{~d}, J=8.3,2 \mathrm{H}), 1.63(\mathrm{~s}$, $3 \mathrm{H}) ;{ }^{13} \mathrm{C}$ NMR (DMSO- $\left.d_{6}, 100 \mathrm{MHz}\right) \delta: 177.14(\mathrm{C}=$ O), $156.69(\mathrm{C}=\mathrm{O}), 139.39(\mathrm{C}), 133.16(\mathrm{C}), 128.89$ $(2 \mathrm{CH}), 127.77(2 \mathrm{CH}), 64.06\left(\mathrm{C}_{\text {spiro }}\right), 25.51\left(\mathrm{CH}_{3}\right)$; IR $\left(\mathrm{KBr} \mathrm{cm}{ }^{-1}\right) \bar{v}: 3274(\mathrm{~N}-\mathrm{H}, \mathrm{s}), 3209(\mathrm{~N}-\mathrm{H}, \mathrm{s})$, $1778(\mathrm{C}=\mathrm{O}, \mathrm{m}), 1725(\mathrm{C}=\mathrm{O}, \mathrm{s}), 1489(\mathrm{C}=\mathrm{C}, \mathrm{w})$, $1447(\mathrm{C}=\mathrm{C}, \mathrm{w}), 1401(\mathrm{~N}-\mathrm{H}, \mathrm{b}, \mathrm{m})$.

2.31 5-Methyl-5-(3-methylphenyl)-imidazolidine-2,4dione $\left(\mathrm{C}_{11} \mathrm{H}_{12} \mathrm{~N}_{2} \mathrm{O}_{2}, \quad 4 \mathrm{l}\right)$ : White powdery; UV $\left(\mathrm{CH}_{3} \mathrm{OH}\right) \quad \lambda_{\max }: 268 \mathrm{~nm} ;{ }^{1} \mathrm{H} \quad \mathrm{NMR}$ (DMSO- $d_{6}$, $400 \mathrm{MHz}) \delta: 10.73\left(\mathrm{~s}, 1 \mathrm{H}, \mathrm{N}_{3}-\mathrm{H}\right), 8.56\left(\mathrm{~s}, 1 \mathrm{H}, \mathrm{N}_{1}-\mathrm{H}\right)$, 7.42-7.25 (s, 3H), 7.12 (s, 1H), 2.30 (s, 3H), 1.87-1.61 $(\mathrm{s}, 3 \mathrm{H}) ;{ }^{13} \mathrm{C}$ NMR (DMSO- $\left.d_{6}, 100 \mathrm{MHz}\right) \delta: 166.16$ $(\mathrm{C}=\mathrm{O}), 157.41(\mathrm{C}=\mathrm{O}), 137.99(\mathrm{C}), 131.30(\mathrm{CH})$, $128.16(\mathrm{CH}), 127.31(\mathrm{CH}), 126.73(\mathrm{CH}), 123.32(\mathrm{C})$, $72.43\left(\mathrm{C}_{\text {spiro }}\right), 22.14\left(\mathrm{CH}_{3}\right), 20.84\left(\mathrm{CH}_{3}\right) ; \mathrm{IR}(\mathrm{KBr}$ $\left.\mathrm{cm}^{-1}\right) \bar{v}: 3290(\mathrm{~N}-\mathrm{H}, \mathrm{s}), 3211(\mathrm{~N}-\mathrm{H}, \mathrm{s}), 3050(=\mathrm{C}-\mathrm{H}$, m), $2972(\mathrm{C}-\mathrm{H}, \mathrm{m}), 1798(\mathrm{C}=\mathrm{O}, \mathrm{m}), 1730(\mathrm{C}=\mathrm{O}, \mathrm{s})$, $1018(\mathrm{~m}), 762(=\mathrm{C}-\mathrm{H}, \mathrm{b})$.

2.3m 5-(3-Chlorophenyl)-5-methyl imidazolidine2,4-dione $\left(\mathrm{C}_{10} \mathrm{H}_{9} \mathrm{~N}_{2} \mathrm{O}_{2} \mathrm{Cl}, 4 \mathrm{~m}\right)$ : White crystal; $\mathrm{UV}\left(\mathrm{CH}_{3} \mathrm{OH}\right) \lambda_{\max }: 270 \mathrm{~nm} ;{ }^{1} \mathrm{H}$ NMR (DMSO- $d_{6}$, $400 \mathrm{MHz}) \delta: 9.86\left(\mathrm{~s}, 1 \mathrm{H}, \mathrm{N}_{3}-\mathrm{H}\right), 7.99\left(\mathrm{~s}, 1 \mathrm{H}, \mathrm{N}_{1}-\right.$ $\mathrm{H}), 7.54-7.47(\mathrm{~m}, 2 \mathrm{H}), 7.18(\mathrm{~d}, J=6.5,1 \mathrm{H}), 7.01$ $(\mathrm{t}, J=6.5,1 \mathrm{H}), 2.09(\mathrm{~s}, 3 \mathrm{H}) ;{ }^{13} \mathrm{C}$ NMR (DMSO- $d_{6}$, $100 \mathrm{MHz}) \delta: 176.4(\mathrm{C}=\mathrm{O}), 158.2(\mathrm{C}=\mathrm{O}), 156.3(\mathrm{C})$, $139.0(\mathrm{CH}), 132.8(\mathrm{CH}), 128.5(\mathrm{CH}), 127.4(\mathrm{CH})$, $126.2(\mathrm{C}), 63.7\left(\mathrm{C}_{\text {spiro }}\right), 25.1\left(\mathrm{CH}_{3}\right)$; IR $\left(\mathrm{KBr} \mathrm{cm}^{-1}\right)$ $\bar{v}$ : $3285(\mathrm{~N}-\mathrm{H}, \mathrm{s}), 3214(\mathrm{~N}-\mathrm{H}, \mathrm{s}), 3042(=\mathrm{C}-\mathrm{H}, \mathrm{m})$,
$2800(\mathrm{C}-\mathrm{H}, \mathrm{m}), 1763(\mathrm{C}=\mathrm{O}, \mathrm{m}), 1716(\mathrm{C}=\mathrm{O}, \mathrm{s})$, $823(\mathrm{~m})$.

2.3n 5,5-Diphenylhydantoin $\left(\mathrm{C}_{15} \mathrm{H}_{12} \mathrm{~N}_{2} \mathrm{O}_{2}, 4 \boldsymbol{n}\right)$ : White needles; UV $\left(\mathrm{CH}_{3} \mathrm{OH}\right) \lambda_{\max }: 276 \mathrm{~nm} ;{ }^{1} \mathrm{H}$ NMR (DMSO- $\left.d_{6}, 400 \mathrm{MHz}\right) \delta: 11.1\left(\mathrm{~s}, 1 \mathrm{H}, \mathrm{N}_{3}-\mathrm{H}\right), 9.33$ $\left(\mathrm{s}, 1 \mathrm{H}, \mathrm{N}_{1}-\mathrm{H}\right), 7.36(\mathrm{~m}, 10 \mathrm{H}) ;{ }^{13} \mathrm{CNMR}$ (DMSO- $d_{6}$, $100 \mathrm{MHz}) \delta: 175.26(\mathrm{C}=\mathrm{O}), 156.43(\mathrm{C}=\mathrm{O}), 140.39$ (2C), $128.96(2 \mathrm{CH}), 128.48(4 \mathrm{CH}), 127.04(4 \mathrm{CH})$, $70.66\left(\mathrm{C}_{\text {spiro }}\right)$; IR $\left(\mathrm{KBr} \mathrm{cm}^{-1}\right) \bar{\nu}: 3270(\mathrm{~N}-\mathrm{H}, \mathrm{s}), 3200$ $(\mathrm{N}-\mathrm{H}, \mathrm{s}), 1770(\mathrm{C}=\mathrm{O}, \mathrm{m}), 1730(\mathrm{C}=\mathrm{O}, \mathrm{s}), 1710$ $\left(\mathrm{C}_{4}=\mathrm{O}_{\text {asym }}, \mathrm{s}\right), 1400(\mathrm{~N}-\mathrm{H}, \mathrm{b}), 760(=\mathrm{C}-\mathrm{H}, \mathrm{b}), 740$ $(=\mathrm{C}-\mathrm{H})$.

2.3o Spiro[fluorene-9,4-imidazolidine]-2,4-dione $\left(\mathrm{C}_{15} \mathrm{H}_{10} \mathrm{~N}_{2} \mathrm{O}_{2}\right.$, 4o $)$ : Yellow powdery; UV $\left(\mathrm{CH}_{3} \mathrm{OH}\right)$ $\lambda_{\max }: 250 \mathrm{~nm} ;{ }^{1} \mathrm{H}$ NMR (DMSO- $\left.d_{6}, 400 \mathrm{MHz}\right) \delta$ : $11.1\left(\mathrm{~s}, 1 \mathrm{H}, \mathrm{N}_{3}-\mathrm{H}\right), 9.33\left(\mathrm{~s}, 1 \mathrm{H}, \mathrm{N}_{1}-\mathrm{H}\right), 7.89-7.85$ $(\mathrm{t}, J=6.7 \mathrm{~Hz}, 2 \mathrm{H}), 7.50-7.45(\mathrm{~m}, 4 \mathrm{H}), 7.37-7.34$ $(\mathrm{t}, J=6.7,2 \mathrm{H}) ;{ }^{13} \mathrm{C}$ NMR (DMSO- $\left.d_{6}, 100 \mathrm{MHz}\right)$ $\delta: 175.26\left(\mathrm{C}_{4}=\mathrm{O}\right), 156.43\left(\mathrm{C}_{2}=\mathrm{O}\right), 140.39(2 \mathrm{C})$, 128.96 (2C), $128.48(4 \mathrm{CH}), 127.04(4 \mathrm{CH}), 70.66$ $\left(\mathrm{C}_{\text {spiro }}\right), \mathrm{IR}\left(\mathrm{KBr} \mathrm{cm}^{-1}\right) \bar{v}: 3270(\mathrm{~N}-\mathrm{H}, \mathrm{s}), 3200(\mathrm{~N}-\mathrm{H}$, s), $1770(\mathrm{C}=\mathrm{O}, \mathrm{m}), 1730\left(\mathrm{C}_{4}=\mathrm{O}, \mathrm{s}\right), 1400(\mathrm{~N}-\mathrm{H}, \mathrm{b}$, w), $760(=\mathrm{C}-\mathrm{H}, \mathrm{b}), 740(=\mathrm{C}-\mathrm{H}, \mathrm{w})$.

\section{Result and discussion}

In this study, we prepared hydantoin derivatives such as 5-phenyl hydantoin (4a), 5-(4-methylphenyl) hydantoin (4b), 5-(4-methoxyphenyl) hydantoin (4c), 5-(2methoxyphenyl) hydantoin (4d), 5-(2-chlorophenyl) hydantoin (4e), 5-(3-pyrrolyl) hydantoin (4f), 5-(2-furryl) hydantoin (4g), 5-isobutyl-5-methyl hydantoin (4h), 1,3-diazaspiro[4,5]decane-2,4-dione (4i), 5-methyl-5phenyl hydantoin (4j), 5-(4-chlorophenyl)-5-methyl hydantoin (4k), 5-methyl-5-(3-methylphenyl) hydantoin (4l), 5-(3-chlorophenyl)-5-methyl hydantoin (4m), 5,5-diphenyl hydantoin (4n) and spiro[fluorene-9,4imidazolidine]-2,4-dione (4o) through the BuchererBergs synthesis in the presence of montmorillonite as a solid catalyst under ultrasound irradiation.

Initially, as shown in table 2, the effect of molar ratio of starting materials on the yield of reaction was studied by considering aldehyde (or ketone): $\mathrm{KCN}$ : $\left(\mathrm{NH}_{4}\right)_{2} \mathrm{CO}_{3}$ ratios and the yield increased from $72 \%$ to $98 \%$, from $1: 1: 5$ to $1: 1.3: 6$. At aldehyde (or ketone): $\mathrm{KCN}:\left(\mathrm{NH}_{4}\right)_{2} \mathrm{CO}_{3}$ ratio of 1:1.3:6, the best yield of 5-isobutyl-5-methyl hydantoin was achieved (table 2). Exceeding ratio of 1:1.3:6 reduced yield of product because of formation of side product. 
Table 2. Effect of molar ratio of starting materials on the yield of reaction. ${ }^{\text {a }}$

\begin{tabular}{|c|c|c|c|c|}
\hline \multicolumn{2}{|c|}{ Weight percentage of product $(\%)$} & \multicolumn{3}{|c|}{ Molar ratio of starting materials (mmol) } \\
\hline Unreacted ketone & 5-isobutyl-5-methyl hydantoin & Ketone & $\mathrm{KCN}$ & $\left(\mathrm{NH}_{4}\right)_{2} \mathrm{CO}_{3}$ \\
\hline 28 & 72 & 1.0 & 1.0 & 5 \\
\hline 2 & 98 & 1.0 & 1.3 & 6 \\
\hline 24 & 84 & 1.0 & 1.5 & 6.2 \\
\hline 23 & 77 & 1.0 & 1.7 & 6.4 \\
\hline 25 & 75 & 1.0 & 2.0 & 6.6 \\
\hline 31 & 69 & 1.0 & 2.1 & 6.8 \\
\hline
\end{tabular}

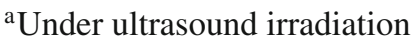

Table 3. Effect of polarity of solvent on the yield of reaction.

\begin{tabular}{lcc}
\hline Solvent & Unreacted ketone & 5-isobutyl-5-methyl-hydantoin (\%) \\
\hline Ethanol & 10 & 90 \\
Methanol & 24 & 76 \\
Methanol: $\mathrm{H}_{2} \mathrm{O}$ & 5 & 95 \\
DMSO & 47 & 53 \\
Ethanol: $\mathrm{H}_{2} \mathrm{O}$ & 2 & 98 \\
Dichloromethane & 69 & 31 \\
\hline
\end{tabular}

Next, we considered the effect of solvent on the yield of reaction. The results in table 3 , show the highest yield of 5,5-disubstituted hydantoin achieved at EtOH to $\mathrm{H}_{2} \mathrm{O}$ ratio of 1:1 (mL). When other solvents were used, no significant improvement in the yield was observed. In the ultrasound-assisted procedure, ethanol was chosen because of solvating power and $\mathrm{H}_{2} \mathrm{O}$ was added for better solvation of $\mathrm{KCN}$ and $\left(\mathrm{NH}_{4}\right)_{2} \mathrm{CO}_{3}$.

Then, we focused on optimizing conditions for synthesis of hydantoin derivatives by using different amounts of clay to determine the effect of the amount of catalyst. The results show that $100 \mathrm{mg}(0.12 \mathrm{mmol})$ is an optimal quantity of catalyst (table 4). The reaction yield with increasing amount of catalyst $(0.22 \mathrm{mmol})$ was not substantially increased.

From the results, optimum reaction conditions were chosen: aldehyde or ketone derivatives (1 mmol), potassium cyanide $(1.3 \mathrm{mmol})$, ammonium carbonate $(6 \mathrm{mmol})$, montmorillonite $(100 \mathrm{mg}, 0.12 \mathrm{mmol})$, ethanol $(10 \mathrm{~mL})$ and $\mathrm{H}_{2} \mathrm{O}(20 \mathrm{~mL})$. Their yields varied from $82 \%$ to $98 \%$ depending on the nature of the $\mathrm{R}^{1}$ and $\mathrm{R}^{2}$ groups.

As shown in table 1, among the various carbonyl compounds it was concluded that more activity can be achieved by aldehydes. Aldehydes (table 1, entry A-G) have shorter reaction time. Also, it is observed that aldehydes (or ketones) containing electron-withdrawing groups were found to be more reactive and could react with $\mathrm{KCN}$ and ammonium carbonate rapidly. In
Table 4. Optimization amount of catalyst for synthesis of 5-isobutyl-5-methyl hydantoin under ultrasound irradiation. ${ }^{\mathrm{a}}$

\begin{tabular}{lccc}
\hline Entry & Clay $(\mathrm{mmol})$ & Time $(\mathrm{min})$ & ${\text { Yield }(\%)^{\mathrm{b}}}^{\mathrm{b}}$ \\
\hline A & 0.0 & 48 & 73 \\
B & 0.09 & 42 & 87 \\
C & 0.13 & 37 & 91 \\
D & 0.18 & 32 & 98 \\
E & 0.22 & 32 & 98 \\
F & 0.25 & 32 & 98
\end{tabular}

${ }^{\mathrm{a}}$ Ketone $(1 \mathrm{mmol}), \mathrm{KCN}(1.3 \mathrm{mmol}),\left(\mathrm{NH}_{4}\right)_{2} \mathrm{CO}_{3}(6 \mathrm{mmol})$

${ }^{\mathrm{b}}$ Isolated yields

contrast, ketones (or aldehydes) containing electrondonating groups have shown lower reactivity. ${ }^{14}$ This shows that the electronic effects of substitutents have a significant effective on the reaction time. The best yield of hydantoins was achieved for dialkyl ketones (table 1, entries $\mathrm{H}$ and I). Finally, the structure of these compounds was investigated using melting point, $\mathrm{UV},{ }^{1} \mathrm{H}$ NMR, ${ }^{13} \mathrm{C}$ NMR and IR.

It is of interest to note that use of the montmorillonite K-10 as solid acid offers high yield of hydantoin derivatives compared to the conventional procedures, probably due to more number molecular interactions. Also, montmorillonite K-10 shows complexation with carbonyl compounds. Therefore, the electrophilic character of carbonyl groups and yield of reactions increase. 

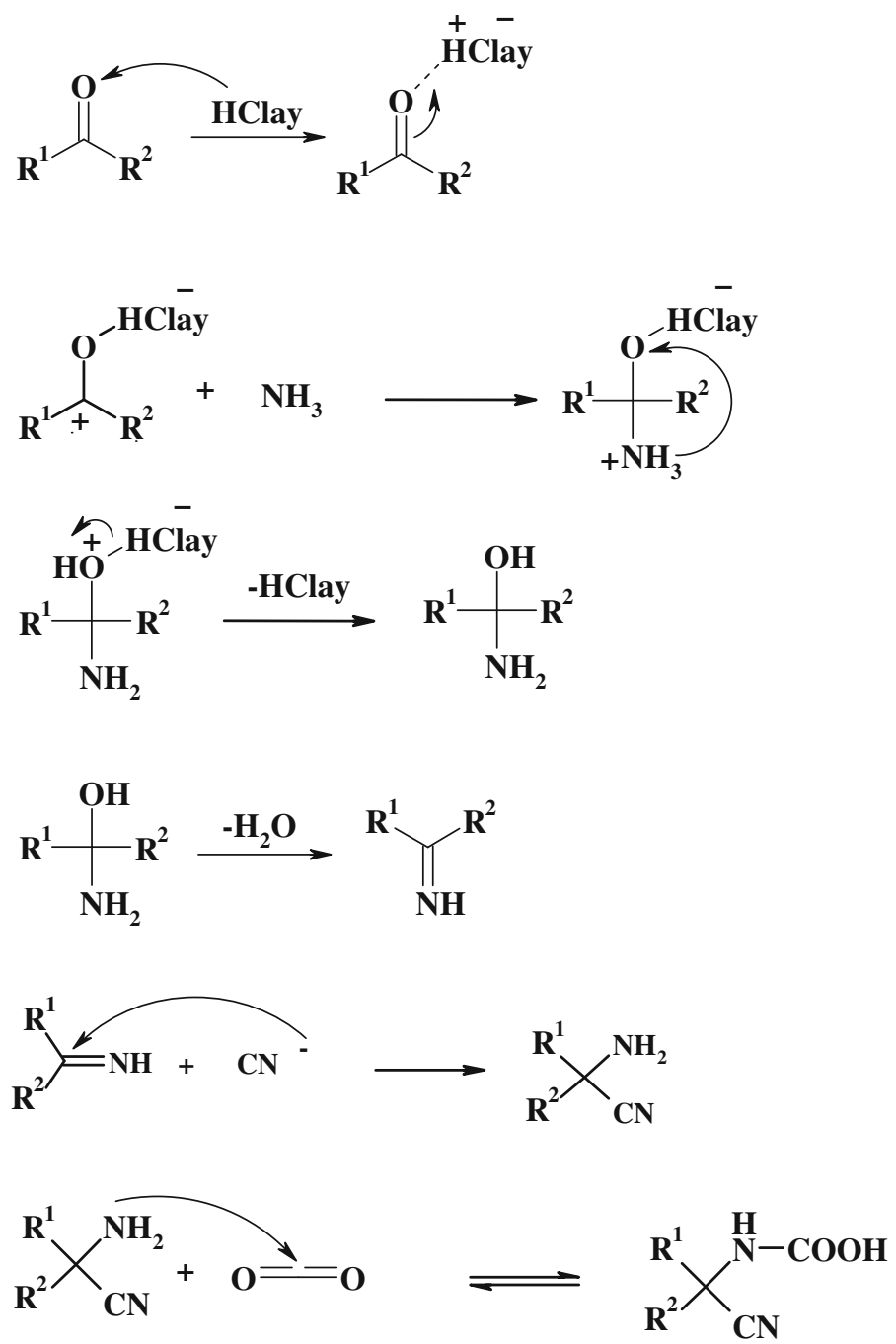<smiles>[R]C([R])(C#N)NC(=O)O</smiles><smiles>[R]C1([R])NC(=O)OC1=N</smiles>

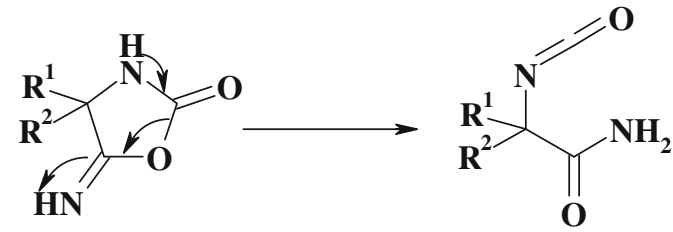<smiles>[R]C([R])(N=C=O)C(N)=O</smiles>

Scheme 2. Possible mechanism for the preparation of hydantoin derivatives. 
A plausible mechanism for this reaction is shown in scheme 2 .

\section{Conclusion}

A facile, one-pot procedure for the synthesis of 5,5disubstituted hydantoins has been developed employing montmorillonite K-10 as a catalyst under ultrasound irradiation as energy source. The K-10 clay is inexpensive and a reusable catalyst. Absorption of ultrasound irradiation in conjunction with the use of montmorillonite provides shorter reaction time, milder reaction condition and higher yield.

\section{Acknowledgements}

The authors gratefully acknowledge financial support from the Research Council of the University of Kashan for supporting this work by Grant No. (159198/XX).

\section{References}

1. Montagne C, Shiers J J and Shipman M 2006 Tetrahedron Lett. 479207

2. Muccioli G G, Poupaert J H, Wouters J, Norberg B, Poppitz W, Scriba G K E and Lambert D M 2003 Tetrahedron $\mathbf{5 9} 1301$

3. Mahmoodi N O and Emadi S 2004 Russ. J. Org. Chem. 40377

4. Zhang D, Ye D, Feng E, Wang J, Shi J, Jiang H and Liu H 2010 J. Org. Chem. 753552

5. Dylag T, Zygmunt M, Maciag D, Handzlik J, Bednarski M, Filipek B and Kiec-Konowicz K 2004 Eur. J. Med. Chem. 391013

6. Basappa C S, Ananda Kumar S, Nanjunda S, Kazuyuki S and Kanchugarakoppal S R 2009 Bioorg. Med. Chem. 174928

7. Zhang W and Lu Y 2003 Org. Lett. 52555

8. Suzen S and Buyukbingol E 2000 Farmaco 55246
9. Mandal A, Krishnan R S G, Thennarasu S, Panigrahi S and Mandal A B 2010 Colloids Surf. B. Bionterfaces 79 136

10. Volonterio A, Ramirez de Arellano C and Zanda M 2005 J. Org. Chem. 702161

11. Gong Y D, Sohn H Y and Kurth M J 1998 J. Org. Chem. 634854

12. Mahmoodi N O and Khodaee Z 2007 Arkivoc 200729

13. Burton S G and Dorrington R A 2004 Tetrahedron: Asymmetry 152737

14. Ahmed S K, Etoga J G, Patel S A and Bridges R J 2011 Bioorg. Med. Chem. Lett. 214358

15. Safari J, Moshtael A N and Ramezan I A 2010 Chin. J. Chem. 28255

16. Moshtael A N and Safari J 2011 Ultrason. Sonochem. 18 640

17. Faghihi Kh and Hagibeygi M 2003 Eur. Polym. J. 39 2307

18. Gallienne E, Muccioli G G, Lambert D M and Shipman M 2008 Tetrahedron Lett. 496495

19. Marvi O, Alizadeh A and Zarrabi S 2011 Bull. Korean Chem. Soc. 324001

20. Bandgar B P, Pandit S S and Sadavarte V S 2001 Green Chem. 3247

21. Shaikh N S, Gajare A S, Deshpande V H and Bedekar A V 2000 Tetrahedron Lett. 41385

22. Li J T, Yin Y and Sun M X 2010 Ultrason. Sonochem. 17363

23. Nabid M R, Tabatabaei S J, Ghahremanzadeh R and Bazgir A 2010 Ultrason. Sonochem. 17159

24. Joshi R S, Mandhane P G, Diwakar S D and Gill C H 2010 Ultrason. Sonochem. 17298

25. Entezari M H, Asghari and Hadizadeh F 2008 Ultrason. Sonochem. 15119

26. Sadjadi S, Sadjadi S and Hekmatshoar R 2010 Ultrason. Sonochem. 17764

27. Henze H R and Speer R J 1942 J. Am. Chem. Soc. 64522

28. Divjak N D, Banjac N R, Valentic N V and Uscumlic G S 2009 J. Serb. Chem. Soc. 741195

29. Li J, Li L, Li T, Li H and Liu J 1996 Ultrason. Sonochem. 3141

30. Faghihi Kh, Zamani Kh and Mallakpour Sh 2002 Iran. Polym. J. 11339 